\title{
On the Structure of the Time-Optimal Path Parameterization Problem with Third-Order Constraints
}

\author{
Hung Pham, Quang-Cuong Pham
}

\begin{abstract}
Finding the Time-Optimal Parameterization of a Path (TOPP) subject to second-order constraints (e.g. acceleration, torque, contact stability, etc.) is an important and wellstudied problem in robotics. In comparison, TOPP subject to third-order constraints (e.g. jerk, torque rate, etc.) has received far less attention and remains largely open. In this paper, we investigate the structure of the TOPP problem with third-order constraints. In particular, we identify two major difficulties: (i) how to smoothly connect optimal profiles, and (ii) how to address singularities, which stop profile integration prematurely. We propose a new algorithm, TOPP3, which addresses these two difficulties and thereby constitutes an important milestone towards an efficient computational solution to TOPP with thirdorder constraints.
\end{abstract}

\section{INTRODUCTION}

Given a robot and a smooth path in the robot's configuration space, the problem of finding the Time-Optimal Parameterization of that Path (TOPP) with second-order constraints (e.g. bounds on acceleration, torques, contact stability) is an important and well-studied in robotics. An efficient algorithm to solve this problem was first proposed in the 1980's [1], [2] and has been continuously perfected since then, see [3] for a historical review. The algorithm has also been extended to handle a wide range of problems, from manipulators subject to torque bounds [1], [4] to vehicles or legged robots subject to balance constraints [5], [6], [7], [8], to kinodynamic motion planning [9], etc.

According to Pontryagin's Maximum Principle, timeoptimal trajectories are bang-bang, which implies instantaneous switches in the second-order quantities that are constrained. For instance, time-optimal trajectories with acceleration constraints will involve acceleration switches, which in turn implies infinite jerk. This is one of the drawbacks of TOPP with second-order constraints.

To address this issue, one can consider TOPP with thirdorder constraints. In many industrial applications, constraining third-order quantities such as jerk, torque rate or force rate is also part of the problem definition [10]. For instance, a hydraulic actuator exerts forces by forcing oil to travel through its piston and gets compressed, which results in the actuating force rate being restricted. As another example, DC electric motors have bounds on input voltages, which translate directly to torque rate constraints.

\section{A. Related works}

While TOPP with second-order constraints can essentially be considered as solved [3], the structure of TOPP with thirdorder constraints is much less well understood. In the sequel, we survey some of the attempts to address TOPP with thirdorder constraints.

1) Numerical integration methods: TOPP with secondorder constraints can be efficiently solved by the numerical integration method: from Pontryagin's Maximum Principle, the optimal velocity profile in the $(s, \dot{s})$ plane $[s(t)$ denotes the position on the path at a given time instant $t$ ] is "bangbang" and can thus be found by integrating successively the maximum and minimum accelerations $\ddot{s}$. In [10], Tarkiainen and Shiller pioneered the extension this approach to thirdorder constraints. Here, one integrates profiles in the $3 D$ space $(s, \dot{s}, \ddot{s})$, following successively maximum, minimum and maximum jerk $\dddot{s}$. As discussed later in Section II] there are two main difficulties: (i) contrary to the $2 \mathrm{D}$ case, minimum and maximum profiles in the $(s, \dot{s}, \ddot{s})$ space do not generally intersect each other, (ii) there are singularities that stop profile integration prematurely. To address (i), the authors proposed to connect the initial maximum jerk profile to the final maximum jerk profile by (a) stepping along the initial profile, (b) at each step, integrate a minimum jerk profile and check whether that profile connects with the final profile. This procedure corresponds to a Single Shooting method, which is known to be sensitive to initial condition [11]. Regarding (ii), the authors did not propose a method to overcome singularities; instead, they suggested to modify the original path to avoid them. However, this workaround is questionable as in practice, paths usually contain a large number of singularities [3].

In a recent work [12], Mattmuller and Gilser proposed an approximate method to compute near-optimal profiles: they introduce "split points" artificially to "guide" the profiles away from singularities. While their method presents the advantage of simplicity, it is sub-optimal and does not help understanding the structure of the true time-optimal solutions.

2) Optimization-based methods: TOPP with second-order constraints can also be solved robustly (albeit at the expense of computation speed [3]) using convex optimization [13], [14]. While we are not aware of any result on formulating TOPP with third-order constraints as a convex optimization problem, there have been a number of works that approximate the constraints to make them convex. For instance, the authors of [15] approximated jerk constraint by linear functions. In [16], the authors replaced jerk constraints by squared jerk terms in the objective function. In a recent work [17], the authors proposed to represent the profiles by a class of $C^{\infty}$ functions. This representation ensures the continuities of higher-order derivatives such as jerk, snap, etc. while still allowing for efficient solutions via convex optimization. 
In [18], [19], [20], the authors approximated the optimal profiles by a piece-wise polynomial in the $(s, \dot{s})$ plane. The polynomials are controlled via a finite set of knot points. The path parameterization problem then becomes equivalent to an optimization problem where the independent variables are the coordinates of the knot points. However, this formulation is non-convex and there is no guarantee that it converges to true time-optimal solutions.

\section{B. Our contributions}

In this paper, we follow the numerical integration approach and investigate the structure of the time-optimal solutions. Specifically, our contribution is threefold:

1) we propose a Multiple Shooting Method (MSM) to smoothly connect two maximum jerk profiles by a minimum jerk profile. This method is more efficient and stable than the the one proposed in [10];

2) we analyze third-order singularities, which arise very frequently and systematically stop profile integration, leading to algorithm failure. We propose a method to address such singularities;

3) based on the above contributions, we implement TOPP3, which solves TOPP with third-order constraints efficiently and yields true optimal solutions in a number of situations.

For simplicity, this paper focuses on pure third-order constraints. Taking into account first- and second-order constraints (e.g. bounds on velocity and acceleration) is possible but will significantly increase the complexity of the exposition.

The remainder of the paper is organized as follows. In Section [I] we introduce the basic notations and formulate the problem. In Section III, we introduce our method to smoothly connect two maximum jerk profiles. In Section IV, we propose a solution to the problem of singularities. In Section V, we present the numerical experiments. Finally, in Section VI, we offer some discussions and directions for future work.

\section{STRUCTURE OF TOPP WITH THIRD-ORDER CONSTRAINTS}

\section{A. Problem setting}

Consider a $n$ dof robotic system whose configuration is a vector $\mathbf{q} \in \mathbb{R}^{n}$. A geometric path $\mathcal{P}$ is a mapping $\mathbf{q}(s)_{s \in\left[0, s_{\text {end }}\right]}$ from $\left[0, s_{\text {end }}\right]$ to the configuration space. A timeparameterization of the path $\mathcal{P}$ is an increasing scalar mapping $s(t)_{t \in[0, T]}$. Differentiating successively $\mathbf{q}(s(t))$ with respect to $t$ yields

$$
\begin{aligned}
\dot{\mathbf{q}} & =\mathbf{q}_{s} \dot{s} \\
\ddot{\mathbf{q}} & =\mathbf{q}_{s s} \dot{s}^{2}+\mathbf{q}_{s} \ddot{s} \\
\dddot{\mathbf{q}} & =\mathbf{q}_{s s s} \dot{s}^{3}+3 \mathbf{q}_{s s} \dot{s} \ddot{s}+\mathbf{q}_{s} \dddot{s},
\end{aligned}
$$

where $\dot{\square}$ denotes time-derivative.

From here on, we shall refer to the first, second and third time-derivatives of the path parameter $s$ as velocity, acceleration and jerk respectively. The time-derivatives of the configuration $\mathbf{q}$ will be called joint velocity, acceleration and jerk.
The boundary conditions of a TOPP with third-order constraints consist of configuration velocities and accelerations at both the start and the goal. Combining with equation (1), one can compute the corresponding initial velocities and accelerations as

$$
s_{0}=0, \quad \dot{s}_{0}=\frac{\left\|\mathbf{v}_{\mathrm{beg}}\right\|}{\left\|\mathbf{q}_{s}(0)\right\|}, \quad \ddot{s}_{0}=\frac{\left\|\mathbf{a}_{\mathrm{beg}}-\mathbf{q}_{s s}(0) \dot{s}_{0}^{2}\right\|}{\left\|\mathbf{q}_{s}(0)\right\|},
$$

where $\mathbf{v}_{\text {beg }}$ and $\mathbf{a}_{\text {beg }}$ are the initial joint velocity and acceleration respectively. The end conditions $\left(s_{1}, \dot{s}_{1}, \ddot{s}_{1}\right)$ can be computed similarly.

We consider third-order constraints of the form

$$
\mathbf{a}(s) \dddot{s}+\mathbf{b}(s) \ddot{s} \ddot{s}+\mathbf{c}(s) \dot{s}^{3}+\mathbf{d}(s) \leq 0,
$$

where $\mathbf{a}(s), \mathbf{b}(s), \mathbf{c}(s), \mathbf{d}(s)$ are $m$-dimensional vectors.

As in the second-order case, the bounds (2) can represent a wide variety of constraints, from direct jerk bounds to bounds on torque rate or force rate 1 . For instance, direct jerk bounds $\left(\mathbf{j}_{\text {min }} \leq \dddot{\mathbf{q}} \leq \mathbf{j}_{\text {max }}\right)$ can be accommodated by setting $\mathbf{a}, \mathbf{b}, \mathbf{c}$, d as follows

$$
\begin{array}{ll}
\mathbf{a}(s)=\left(\begin{array}{c}
\mathbf{q}_{s}(s) \\
-\mathbf{q}_{s}(s)
\end{array}\right), & \mathbf{b}(s)=\left(\begin{array}{c}
3 \mathbf{q}_{s s}(s) \\
-3 \mathbf{q}_{s s}(s)
\end{array}\right), \\
\mathbf{c}(s)=\left(\begin{array}{c}
\mathbf{q}_{s s s}(s) \\
-\mathbf{q}_{s s s}(s)
\end{array}\right), & \mathbf{d}(s)=\left(\begin{array}{c}
-\mathbf{j}_{\max } \\
\mathbf{j}_{\min }
\end{array}\right) .
\end{array}
$$

Similar to the classical TOPP algorithm with second order constraints [1], [3], one can next define, at any state $(s, \dot{s}, \ddot{s})$, the minimum jerk $\gamma(s, \dot{s}, \ddot{s})$ and maximum jerk $\eta(s, \dot{s}, \ddot{s})$ as follows

$$
\begin{aligned}
& \gamma(s, \dot{s}, \ddot{s}):=\max _{i}\left\{\frac{-b_{i}(s) \dot{s} \ddot{s}-c_{i}(s) \dot{s}^{3}-d_{i}(s)}{a_{i}(s)} \mid a_{i}(s)<0\right\}, \\
& \eta(s, \dot{s}, \ddot{s}):=\min _{i}\left\{\frac{-b_{i}(s) \dot{s} \ddot{s}-c_{i}(s) \dot{s}^{3}-d_{i}(s)}{a_{i}(s)} \mid a_{i}(s)>0\right\} .
\end{aligned}
$$

Note that the above definitions neglect the case where some $a_{i}$ are zero. As in the case of second-order constraints, a path position $s$ where at least one of the $a_{i}(s)$ is zero can trigger a singularity.

A profile is is a curve in the $(s, \dot{s}, \ddot{s})$-space where $s$ is always increasing. A time-parameterization of $\mathbf{q}$ corresponds to a profile connecting $\left(s_{0}, \dot{s}_{0}, \ddot{s}_{0}\right)$ to $\left(s_{1}, \dot{s}_{1}, \ddot{s}_{1}\right)$. From the definition of $\gamma$ and $\eta$, a time-parameterization of $\mathbf{q}$ satisfies the constraints 2 if and only if the jerk $\dddot{s}$ along the corresponding profile satisfies $\gamma \leq \dddot{s} \leq \eta$.

Next, we define a minimum jerk (resp. maximum jerk) profile as a profile along which $\dddot{s}=\gamma(s, \dot{s}, \ddot{s})$ (resp. $\dddot{s}=$ $\eta(s, \dot{s}, \ddot{s}))$. From Pontryagin's Maximum Principle, the timeoptimal profile follows successively maximum and minimum jerk profiles [10]. Finding which profiles to follow and when to switch between two consecutive profiles is therefore the fundamental issue underlying TOPP with third-order constraints.

\footnotetext{
${ }^{1}$ In fact, torque rate and force rate involve an additional term $\dot{s} \mathbf{e}(s)$, but their treatment is not fundamentally different from what is presented in this paper.
} 


\section{B. Introducing TOPP3}

If there is no singularity, the optimal profile has a max-minmax structure [10]. Thus, to find the optimal profile, one can proceed as follows, see Fig. 1 for an illustration

1) integrate forward the maximum jerk profile (following $\eta)$ from $\left(s_{0}, \dot{s}_{0}, \ddot{s}_{0}\right)$;

2) integrate backward the maximum jerk profile (following $\eta)$ from $\left(s_{1}, \dot{s}_{1}, \ddot{s}_{1}\right)$

3) find a minimum jerk profile that starts from one point on the first profile and ends at one point on the second one.

All profiles are integrated until failure unless otherwise specified.

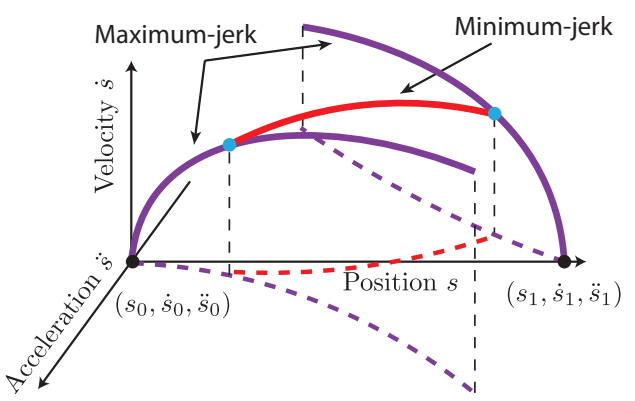

Figure 1. An optimal parametrization with max-min-max structure.

To find the connecting profile of step (3), Tarkiainen and Shiller proposed to step along the first profile, integrate following minimum jerk and check whether it connects to the second profile. This exhaustive procedure is computationally inefficient and numerically unstable. We propose, in Section III] a more efficient way (bridge) to perform the connection.

In the presence of singularities $\left(a_{i}(s)=0\right)$, the max-minmax procedure just described will fail, irrespective of the connection method. This is because the integrated profiles diverge as they approach a singularity and quickly terminate, see Fig. 3 3 A. This behavior is the same as in the second-order case [3]. To address this issue, we propose, in Section IV] a method (extend) that allows extending profiles through singularities. Note that the structure of the final profile will no longer be max-min-max but max-min-max-...-min-max.

The above discussion can be encapsulated into Algorithm 1 . which we call TOPP3. In the next sections, we shall discuss in detail the two main components of TOPP3, namely bridge and extend.

\section{Remarks}

1) Optimality of the connection: In the absence of singularities, the max-min-max structure described earlier is only a necessary condition for optimality and not a sufficient one. Theoretically, to find the true optimal solution, it suffices to enumerate all candidates having max-min-max structure and select the one with the shortest time duration.

Next, since the first and last maximum jerk profiles are welldetermined (their starting (resp. ending) conditions are given),

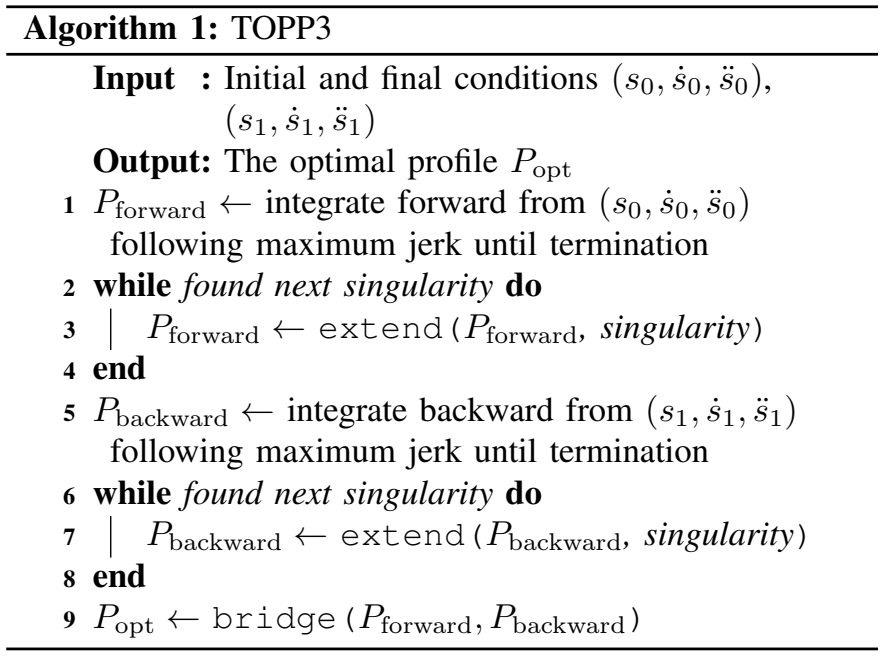

different solutions only differ by the connecting minimum jerk profile. If there exists one unique minimum jerk profile that can connect the two maximum jerk profiles, then the corresponding solution is automatically the optimal one. In practice, we have never encountered the case when there are more than one possible connecting minimum jerk profile. Note that Tarkiainen and Shiller [10] implicitly assumed that there exists one unique connecting minimum jerk profile.

2) Other switching structures: The TOPP problem with second-order constraints involves three types of switch points, i.e., points where the optimal profile changes from minimum acceleration to maximum acceleration and vice-versa (cf. [3]): (a) singular (b) discontinuous and (c) tangent points. These switch points, in general, lie on the Maximum Velocity Curve (MVC).

We argue that TOPP with third-order constraints similarly involves singular, discontinuous and tangent switch points, and that those points, in general, lie on the Maximum and Minimum Acceleration Surfaces (MaAS and MiAS).

In Section IV] we shall discuss the singular switch points, which are the most commonly encountered and harmful (if inappropriately treated) switch points. As in the case of secondorder constraints, discontinuous switch points can always be avoided if the path is sufficiently smooth. Finally, tangent switch points are left for future work. Let us simply note here that they occur much less frequently than singular switch points.

Another type of switching structure arises when constraints of different orders interact. For instance, in the classic TOPP problem, when an integrated profile hits a first-order constraint (e.g. direct velocity bound), then it must "slide" along the boundary defined by that first-order constraint, giving rise to a different type of switch point. We envisage that such switching behavior can also happen when first, second, and third-order constraints interact. The study of these interactions is also left for future work. 


\section{Connecting PRofiles Using Multiple Shooting}

Instead of the exhaustive search suggested in [10], we propose here a method, termed bridge, which is based on Multiple Shooting to find a minimum jerk profile that can connect two maximum jerk profiles. Specifically, consider the problem of connecting two maximum jerk profiles $A$ and $B$. We define a potential solution $\mathbf{x}$ as a $(2 N+4)$-dimensional vector

$$
\mathbf{x}:=\left[\dot{s}_{0}, \ddot{s}_{0}, \ldots, \dot{s}_{N}, \ddot{s}_{N}, s_{A}, s_{B}\right],
$$

where $\dot{s}_{i}, \ddot{s}_{i}, i \in[0, N]$ are the guessed velocities and accelerations at the $i$-th point and $\left(s_{A}, s_{B}\right)$ are the guessed starting and ending positions on profiles $A$ and $B$ respectively. We consider a uniform grid, i.e.

$$
s_{i}:=s_{A}+\frac{i}{N}\left(s_{B}-s_{A}\right) .
$$

Next, assume that we integrate following minimum jerk from $\left(s_{i}, \dot{s}_{i}, \ddot{s}_{i}\right)$ until $s_{j}$. We define the function $X: \mathbb{R}^{4} \rightarrow \mathbb{R}^{2}$ as

$$
X\left(s_{i}, \dot{s}_{i}, \ddot{s}_{i}, s_{j}\right):=\left(\dot{s}_{j}, \ddot{s}_{j}\right),
$$

where $\dot{s}_{j}, \ddot{s}_{j}$ are the corresponding velocity and acceleration at $s_{j}$. This allows us to define the defect function by

$$
F(\mathbf{x}):=\left(\begin{array}{c}
X\left(s_{0}, \dot{s}_{0}, \ddot{s}_{0}, s_{1}\right)-\left[\dot{s}_{1}, \ddot{s}_{1}\right]^{T} \\
X\left(s_{1}, \dot{s}_{1}, \ddot{s}_{1}, s_{2}\right)-\left[\dot{s}_{2}, \ddot{s}_{2}\right]^{T} \\
\ldots \\
X\left(s_{N-1}, \dot{s}_{N-1}, \ddot{s}_{N-1}, s_{N}\right)-\left[\dot{s}_{N}, \ddot{s}_{N}\right]^{T} \\
r_{\mathrm{A}}\left(s_{\mathrm{A}}\right)-\left[\dot{s}_{0}, \ddot{s}_{0}\right]^{T} \\
r_{\mathrm{B}}\left(s_{\mathrm{B}}\right)-\left[\dot{s}_{N}, \ddot{s}_{N}\right]^{T}
\end{array}\right),
$$

where $r_{\mathrm{A}}\left(s_{\mathrm{A}}\right)$ (resp. $\left.r_{\mathrm{B}}\left(s_{\mathrm{B}}\right)\right)$ are the velocity and acceleration on profile A (resp. B) at position $s_{\mathrm{A}}$ (resp. $s_{\mathrm{B}}$ ).

The connection problem can now be formulated as

$$
\begin{aligned}
\text { solve } & F(\mathbf{x})=0 \\
\text { subject to } & s_{\text {Abeg }} \leq s_{\mathrm{A}} \leq s_{\text {Aend }}, \\
& s_{\text {Bbeg }} \leq s_{\mathrm{B}} \leq s_{\text {Bend }}
\end{aligned}
$$

where $\left(s_{\text {Abeg }}, s_{\text {Aend }}\right)$ denote positions of profile A's end points (resp. profile B).

To solve (5), we employ the Newton method. Although the problem is non-linear and non-convex, the algorithm still converges very quickly to a solution. Fig. 2 shows a particular instance where a solution can be found in 4 iterations.

Regarding computational cost, we found that it correlates to the magnitude of jerk bounds. For instance, at $1000 \mathrm{rads}^{-3} \mathrm{a}$ bridge function call takes only $10 \mathrm{~ms}$ while at $100 \mathrm{rads}^{-3}$, it is approximately $200 \mathrm{~ms}$. How jerk bounds precisely affect computation time is left for future investigations.

\section{CharacterizING AND ADDRESSING SINGUlarities}

Singularities in the third-order case are very similar to those in the second-order case: (i) they arise at positions $s$ where the minimum and maximum jerk $\gamma$ and $\eta$ cannot be properly defined because of the division by $a_{k}(s)=0$ for some $k$; (ii) they cause profiles to terminate prematurely, causing algorithm failure (see Fig. 3). In the sequel, we discuss how
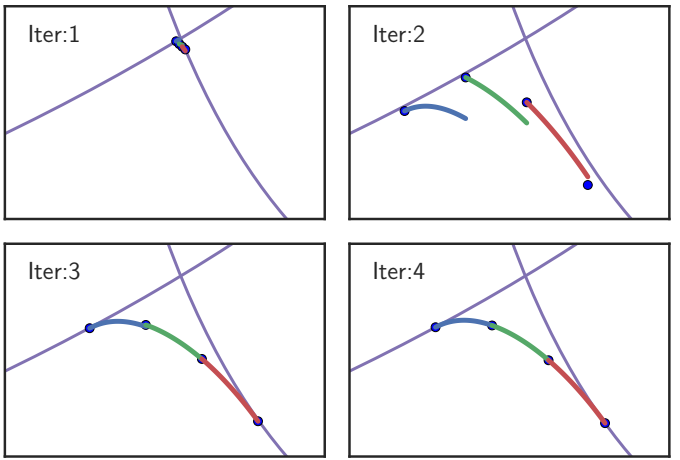

Figure 2. Using MSM to find a minimum jerk profile connecting two maximum jerk profiles (purple). Note that the profiles are in 3D but projected to $2 \mathrm{D}$ for convenience. The number of segments is $N=3$. The algorithm took 4 iterations to converge.

to characterize third-order singularities and how to address singularities, taking much inspiration from the second-order case [3].

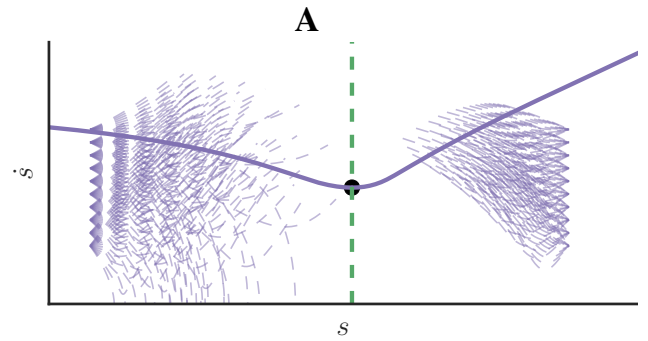

B

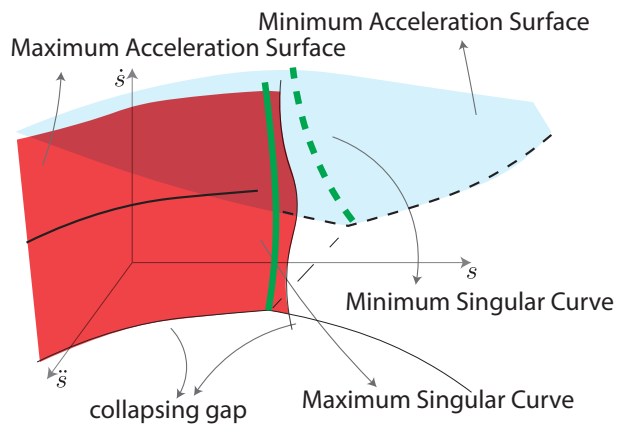

Figure 3. A: Profiles approaching singularities (purple dashed lines) diverge and terminate early. The diverging directions include both vertical (along $\dot{s}$ axis) and lateral (along $\ddot{s}$ axis, which is not shown). However, profiles (purple solid lines) starting from a point lying between the singular curves (green dashed lines) are not affected by the singularities. B: Singularities consist of maximum or minimum singular curves lying on the maximum or minimum acceleration surfaces (MaAS/MiAS) respectively.

\section{A. Maximum and Minimum Acceleration Surfaces}

In the second-order case, singularities mostly appear on the Maximum Velocity Curve (MVC) [3], [21], [22]. Here, we define the Maximum and Minimum Acceleration Surfaces (MaAS and MiAS), which are the third-order counterparts of the MVC. 
Definition 1 (MaAS/MiAS). Consider the set of feasible accelerations

$$
\mathcal{F}(s, \dot{s}):=\left\{\ddot{s} \mid \exists \dddot{s}, \mathbf{a}(s) \dddot{s}+\mathbf{b}(s) \ddot{s} \ddot{s}+\mathbf{c}(s) \dot{s}^{3}+\mathbf{d}(s) \leq 0\right\} .
$$

We define the MaAS and MiAS by

$$
\begin{aligned}
& \operatorname{MaAS}(\mathrm{s}, \dot{\mathrm{s}}):=\max _{\ddot{s} \in \mathcal{F}(s, \dot{s})} \ddot{s} \\
& \operatorname{MaAS}(\mathrm{s}, \dot{\mathrm{s}}):=\min _{\ddot{s} \in \mathcal{F}(s, \dot{s})} \ddot{s} .
\end{aligned}
$$

If $\mathcal{F}(s, \dot{s})$ is empty then the surfaces are not defined at $(s, \dot{s})$.

This definition does not use the maximal controls $\gamma$ and $\eta$ but directly uses the constraints (2). The advantage is that even in the presence of a constraint $k$ such that $a_{k}(s)=0$, the surfaces are still well-defined. Additionally, on both surfaces, the maximum and minimum jerks are equal almost everywhere except on singular curves (see Prop. 2 in the Appendix).

\section{B. Characterizing third-order singularities}

Definition 2 (Singular curve). We say that the $k$-th constraint triggers a singularity at $s^{*}$ if $a_{k}\left(s^{*}\right)=0$ and the set $C$ defined by

$$
\left.\begin{array}{rl}
C & :=\left\{\left(s^{*}, \dot{s}, \ddot{s}\right) \mid \exists \dddot{s}:\right. \\
& a_{k}\left(s^{*}\right) \dddot{s}+b_{k}\left(s^{*}\right) \dot{s} \ddot{s}+c_{k}\left(s^{*}\right) \dot{s}^{3}+d_{k}\left(s^{*}\right)=0, \\
& a_{i}\left(s^{*}\right) \dddot{s}+b_{i}\left(s^{*}\right) \ddot{s} \ddot{s}+c_{i}\left(s^{*}\right) \dot{s}^{3}+d_{i}\left(s^{*}\right) \leq 0, i \neq k
\end{array}\right\}
$$

is non-empty. We say $C$ is the singular curve at $s^{*}$.

We show in the Appendix that all singular curves lie on either the MaAS or MiAS (Prop. 11). Furthermore, a singular curve lies on the MaAS if $b_{k}\left(s^{*}\right)>0$ and on the MiAS if $b_{k}\left(s^{*}\right)<0$. We shall also refer to singular curves on MaAS and MiAS as maximum and minimum singular curves respectively.

As in the second-order case, we can note the following behaviors:

- Forward integrations following maximum jerk and backward integrations following minimum jerk diverge when they approach a minimum singular curve;

- Forward integrations following minimum jerk and backward integrations following maximum jerk diverge when they approach a maximum singular curve;

- Forward and backward integrations following either maximum jerk or minimum jerk starting from a feasible point at $s^{*}$ are not affected by the singular curves.

To understand these observations, we note that at fixed velocity $\dot{s}=\dot{s}_{0}$, constraints (7) has the same form as second orderconstraints

$$
\mathbf{a}_{2 \text { nd }}(s) \ddot{s}+\mathbf{b}_{2 \text { nd }}(s) \dot{s}^{2}+\mathbf{c}_{2 \text { nd }}(s) \leq 0,
$$

where $\mathbf{a}_{2 \text { nd }}, \mathbf{b}_{2 \text { nd }}, \mathbf{c}_{2 \text { nd }}$ are the corresponding vectors. In the second-order case, constraints (8) cause integrations to diverge. It therefore suggests that integrations (third-order) projected on to a fixed velocity surface would likely diverge, which is consistent with our observations. A more rigorous analysis is left for future work.

To compute a singular curve $C$ at $s^{*}$, we simply find the maximum and minimum velocities $\dot{s}_{\max }^{*}, \dot{s}_{\min }^{*}$ of each curve and compute $\ddot{s}^{*}$ by the equality constraint in Eq. (7). This procedure correctly returns $C$ because a singular curve is connected (See Prop. 3 in the Appendix).

To compute $\dot{s}_{\max }^{*}, \dot{s}_{\min }^{*}$ one needs to solve a pair of linear programming problems

$$
\begin{aligned}
\text { maximize } & {[0,0,1]^{T}\left[\dddot{s}, \dot{s} \ddot{s}, \dot{s}^{3}\right] } \\
\text { subject to } & 7
\end{aligned} \text { and } \begin{aligned}
& \\
& \text { maximize } {[0,0,-1]^{T}\left[\dddot{s}, \dot{s} \ddot{s}, \dot{s}^{3}\right] } \\
& \text { subject to }(7) .
\end{aligned}
$$

C. Extending a profile through a singularity

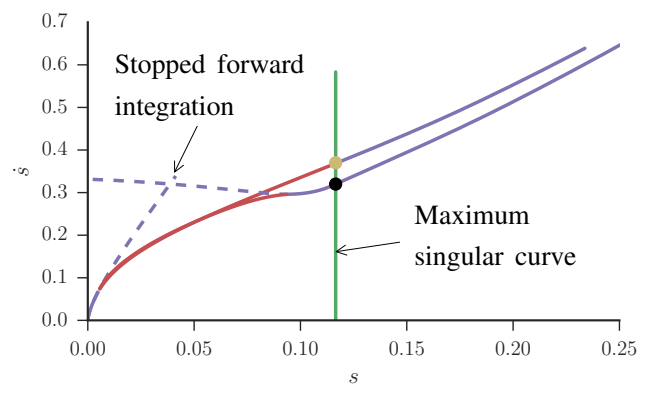

Figure 4. Comparison of the conjectured time-optimal extension (via the yellow point) with an extension computed by picking a feasible point lying at $s^{*}$ (black point) and uses it to extend the forward integration. Note that the profiles are projected onto the $(s, \dot{s})$ plane.

We now describe a method, termed extend, to extend a forward maximum jerk profile through a singularity $s^{*}$. As noted before, integrating forward following maximum jerk from $s^{*}$ is not problematic, so the main difficulty consists of connecting backwards to the initial forward profile.

Note first that there exists a family of possible backward connections, obtained as below

1) choose a feasible point at $s^{*}$ (i.e. a point $\left(s^{*}, \dot{s}, \ddot{s}\right)$ for which there exists a feasible jerk);

2) integrate backward following maximum jerk from this point;

3) connect the original forward maximum jerk profile to the new backward maximum jerk profile by the bridge procedure of Section III]

We conjecture that, within this family of possible connections, the optimal connection has the following properties

1) the starting point at $s^{*}$ belongs to the maximum singular curve;

2) there is no backward maximum jerk profile: in other words, the connecting minimum jerk profile starts directly at $s^{*}$;

3) as a consequence, the min-to-max switch happens directly at $s^{*}$ and not before, as in the generic case above. 
Table I

KINEMATIC LIMITS FOR DENSO ROBOT ARM

\begin{tabular}{lrrrrrr}
\hline Limits & J1 & J2 & J3 & J4 & J5 & J6 \\
\hline Vel $\left(\mathrm{rads}^{-1}\right)$ & 3.92 & 2.61 & 2.85 & 3.92 & 3.02 & 6.58 \\
Accel $\left(\mathrm{rads}^{-2}\right)$ & 19.7 & 16.8 & 20.7 & 20.9 & 23.7 & 33.5 \\
Jerk $\left(\mathrm{rads}^{-3}\right)$ & 100. & 100. & 100. & 100. & 100. & 100. \\
\hline
\end{tabular}

See Fig. 4 for an illustration.

Accordingly, we propose the following strategy to perform the backward connection using MSM. Using subscript A and $\mathrm{C}$ to denote the forward profile and the singular curve, we define a solution $\mathbf{x} \in \mathbb{R}^{2 N+4}$ as

$$
\mathbf{x}:=\left[\dot{s}_{0}, \ddot{s}_{0}, \ldots, \dot{s}_{N}, \ddot{s}_{N}, s_{\mathrm{A}}, \dot{s}_{\mathrm{C}}\right],
$$

where $\dot{s}_{i}$ and $\ddot{s}_{i}$ are the velocity and acceleration at $s_{i}, s_{\mathrm{A}}$ and $\dot{s}_{\mathrm{C}}$ are the guessed starting position and ending velocity on $\mathrm{A}$ and $\mathrm{C}$ respectively. This gives the defect function

$$
F(\mathbf{x}):=\left(\begin{array}{c}
X\left(s_{1}, \dot{s}_{1}, \ddot{s}_{1}, s_{0}\right)-\left[\dot{s}_{0}, \ddot{s}_{0}\right]^{T} \\
X\left(s_{2}, \dot{s}_{2}, \ddot{s}_{2}, s_{1}\right)-\left[\dot{s}_{1}, \ddot{s}_{1}\right]^{T} \\
\ldots \\
X\left(s_{N}, \dot{s}_{N}, \ddot{s}_{N}, s_{N-1}\right)-\left[\dot{s}_{N-1}, \ddot{s}_{N-1}\right]^{T} \\
r_{\mathrm{A}}\left(s_{A}\right)-\left[\dot{s}_{0}, \ddot{s}_{0}\right]^{T} \\
\bar{r}_{\mathrm{C}}\left(\dot{s}_{\mathrm{C}}\right)-\left[\dot{s}_{N}, \ddot{s}_{N}\right]^{T}
\end{array}\right),
$$

where $r_{\mathrm{A}}\left(s_{\mathrm{A}}\right)$ and $\bar{r}_{\mathrm{C}}\left(s_{\mathrm{C}}\right)$ give the velocity and acceleration on the profile and the curve. Again, Newton's method can be employed to find the root of 9 .

There exists an additional difficulty with respect to Section III: the optimal jerk is ill-defined on the singular curves because of a division by zero: $a_{k}\left(s^{*}\right)=0$. Taking again inspiration from [3], we can show that the optimal jerk on the singular curves is in fact given by the following singular jerk

$\dddot{s}_{\mathrm{sglr}}=-\frac{d_{k}^{\prime}\left(s^{*}\right)+c_{k}^{\prime}\left(s^{*}\right) \dot{s}^{3}+3 c_{k}\left(s^{*}\right) \dot{s} \ddot{s}+b\left(s^{*}\right)\left(\ddot{s} \ddot{s}+\ddot{s}^{2} / \dot{s}\right)}{a_{k}^{\prime}\left(s^{*}\right)+b_{k}\left(s^{*}\right)}$.

This expression can be derived similarly as in [3].

Fig. (4) compares the conjectured optimal connection with a generic connection. One can note that the conjectured profile has a higher velocity at any position, which implies timeoptimality.

The case of a backward extension can be treated similarly.

\section{NUMERICAL EXPERIMENTS}

\section{A. Simulation results}

We implemented and tested TOPP 3 on a random geometric path subjecting to constraints on joint velocities, accelerations and jerks (Table I). Implementation of acceleration and velocity constraints follows [3].

Different scenarios were considered. In the first one, we set restrictive bounds on joint jerks at $100 \mathrm{rad} / \mathrm{s}^{3}$. There were several singularities which are plotted as green lines in Fig. 5. Despite the existence of these singularities, TOPP3 was able
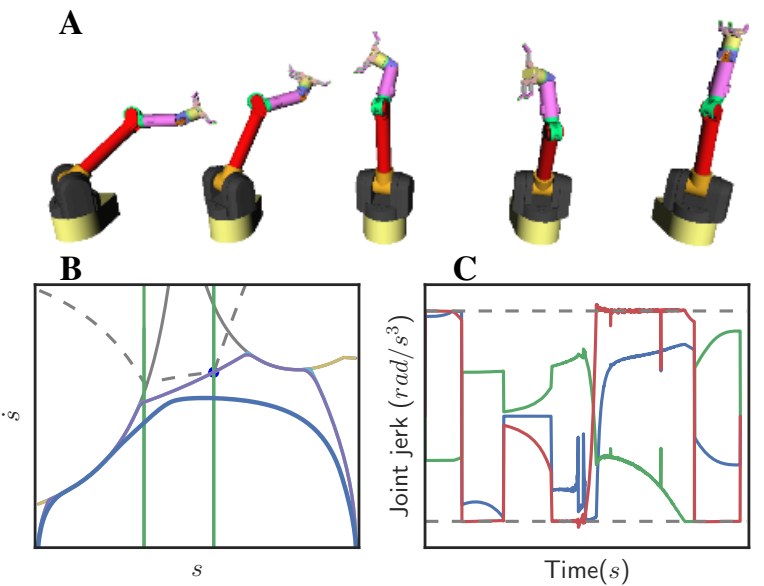

Figure 5. A: Snapshots of the robot. B: The profile constrained at $100 \mathrm{rad} / \mathrm{s}^{3}$ (blue), the profile constrained at $1000 \mathrm{rad} / \mathrm{s}^{3}$ (purple) and the profile with unconstrained jerk (light blue). Singularities are plotted as green lines. Note that the profiles are projected onto the $(s, \dot{s})$ plane. C: Joint jerks versus time.

to compute the time-optimal parametrization. We note that TOPP3 extended the forward profile once via the maximum singular curve on the left.

Next, we considered a more practical set of bounds at $1000 \mathrm{rad} / \mathrm{s}^{3}$ on joint jerks. In this case, TOPP 3 also terminated successfully. Moreover, we found that singularities did not affect the profiles: the final profile was computed without having to extend any profile via any singularities.

Lastly, we compared these profiles with one that does not subject to bounds on joint jerks (computed with the original TOPP algorithm). We observed that the profile bounded at $100 \mathrm{rad} / \mathrm{s}^{3}$ appears to be a smoothed version of the profile bounded at $1000 \mathrm{rad} / \mathrm{s}^{3}$, which in turn is a smoothed version of the one not subject to any jerk bound (Fig. 5).

Fig. 6 shows the joint trajectories computed from these profiles. We note that the profiles are bang-bang, satisfy all kinematic constraints and have different total duration. In particular, they last $1.36 \mathrm{sec}, 1.19 \mathrm{sec}$ and $1.18 \mathrm{sec}$. Note that, when there is no bound on jerk, the latter reaches extremely high values (Fig. 6-C3).

Table III reports computation time for the three scenarios respectively. The experiments were ran on a single core at $2.00 \mathrm{GHz}$ and $8 \mathrm{~Gb}$ of memory. We remark that the preprocessing - computation of singularities and switch points - is written in Python and is significantly slower than the integration and MSM procedures which are written in Cython. Therefore, we only compared the total time* which neglects pre-processing. In this regard, computing the first and second profiles took 83 times and 19 times longer than the third one.

\section{B. Singularities caused by third-order constraints}

Third-order singularities appear quite frequently, almost as frequent as singular switch points which are many [3]. In the Fig. 5, one can see that the singular curves (green lines) 

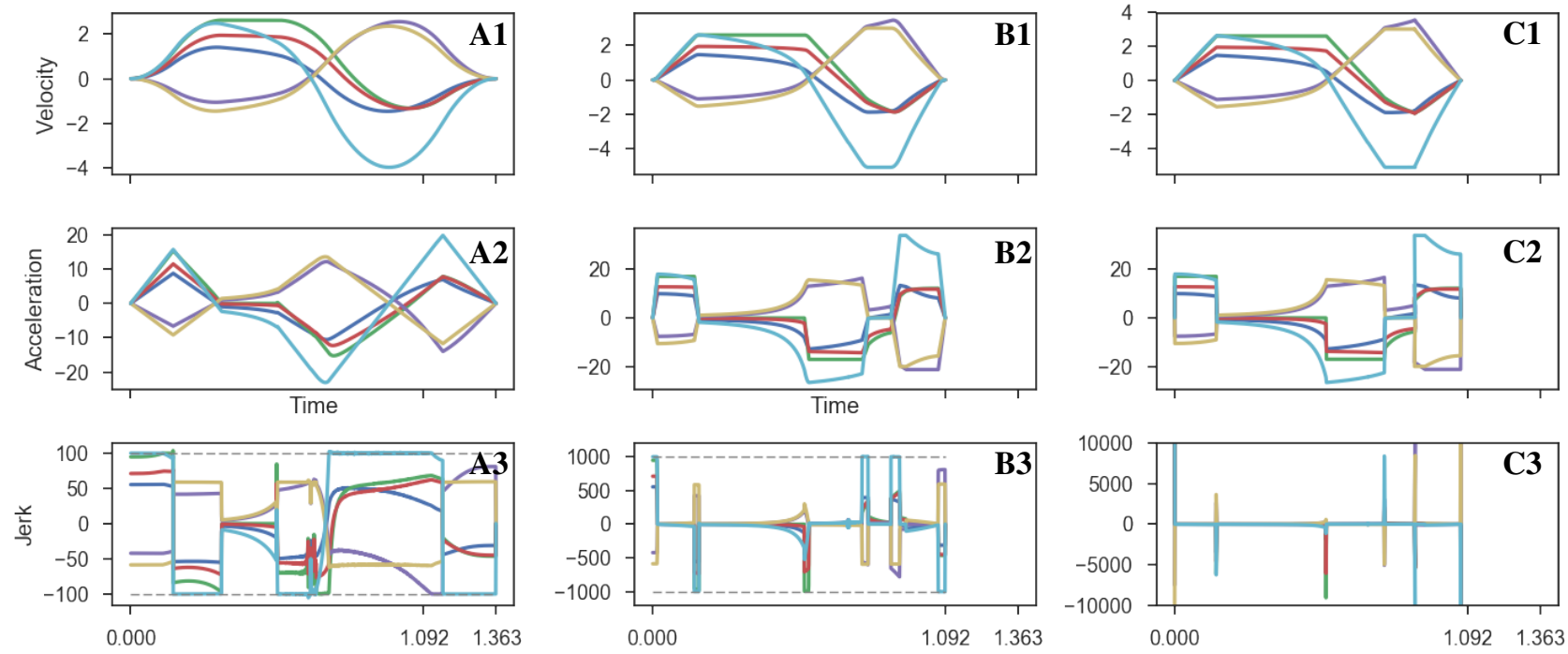

Figure 6. Comparing the resulting trajectories from TOPP3 with $100 \mathrm{rad} / \mathrm{s}^{3}$ bound on joint jerks (A1-3), 1000 rad/ $\mathrm{s}^{3}$ bound on joint jerks (B1-3) and one from TOPP without any bound on jerk (C1-3). Note that, when there is no bound on jerk, the latter reaches extremely high values $(\mathrm{C} 3)$.

Table II

COMPUTATION TIME FOR DIFFERENT LEVELS OF BOUND ON JOINT JERKS

\begin{tabular}{lrrr}
\hline Jerk bound $\left(\mathrm{rad} / \mathrm{s}^{-3}\right)$ & 100 & 1000 & $\infty$ \\
\hline Time step (ms) & 1 & 1 & 1 \\
Total (ms) & 754 & 181 & 72 \\
$\quad$ integrate & 4.05 & 2.36 & 2.24 \\
bridge & 364 & 42.2 & 0.3 \\
extend & 134 & 73.2 & 3.50 \\
Pre-process & 243 & 63.7 & 65.9 \\
Total ${ }^{2}(\mathrm{~ms})$ & 511 & 118 & 6.1 \\
& $83 \mathrm{x}$ & $19 \mathrm{x}$ & $1 \mathrm{x}$ \\
\hline
\end{tabular}

correspond to points where the MVC is continuous but is not differentiable, which are singular switch points [3].

We observed that in the second scenario $\left(1000 \mathrm{rad} / \mathrm{s}^{3}\right)$, singularities did not affect integrations. We found that this phenomenon only appears when the bounds on joint jerks are high in comparison with the bounds on joint accelerations. Here is one possible explanation: at high jerk bounds, the singular curves have high acceleration. However, as second-order constraints restrict integrations from having high acceleration, the integrations will not come close to these curves. Now, as analyzed, we observe that integrations only diverge near to the singular curves; therefore, these integrations are not affected. The precise conditions at which this happens is left for future work.

\section{CONCLUSION}

In this paper, we have studied the structure of the TimeOptimal Path Parameterization (TOPP) problem with thirdorder constraints. We have argued that the optimal profile can

\footnotetext{
${ }^{2}$ Not including time to compute singularities and switch points.
}

be obtained by integrating alternatively maximum and minimum jerk, yielding a max-min-max-...-min-max structure. We have identified two main difficulties in the integration process: (i) how to smoothly connect two maximum jerk profiles by a minimum jerk profile, and (ii) how to extend the integration through singularities which, if not properly addressed, would systematically cause integration failure. We proposed some solutions to these two difficulties and, based on these solutions, implemented an algorithm - TOPP3 - to solve the TOPP problem with third-order constraints in a number of representative scenarios.

There are still a number of open theoretical and practical questions, which we are currently actively investigating. For instance,

1) Under which condition the profile returned by the connection procedure (bridge) is unique? Alternatively, can we enumerate all connecting profiles?

2) Similarly, under which condition the profile returned by the extension procedure (extend) is unique?

3) How to characterize and address the tangent switch points (which probably exist in the form of tangent curves)?

Addressing all these (difficult) questions will enable us to implement TOPP3 in a fast and robust manner, which in turn can be useful for a wide range of robotics applications.

\section{APPENDIX}

\section{A. Some proofs regarding third-order singularities}

Proposition 1. All singular curve lies on either the MaAS or the MiAS. Furthermore, a singular curve lies on the MaAS if $b_{k}\left(s^{*}\right)>0$ and on the MiAS if $b_{k}\left(s^{*}\right)<0$.

We assume $b_{k}\left(s^{*}\right) \neq 0$ since it is fairly rare for both $a_{k}$ and $b_{k}$ to become zero. 
Proof. We will prove the first proposition by contradiction. Consider a singular curve $C$ triggered by the $k$-th constraint, there exists a point $\left(s^{*}, \dot{s}^{*}, \ddot{s}^{*}\right) \in C$ that do not lie on either the MaAS or MiAS. That is

$$
\operatorname{MaAS}\left(\mathrm{s}^{*}, \dot{\mathrm{s}}^{*}\right)<\ddot{\mathrm{s}}^{*}<\operatorname{MaAS}\left(\mathrm{s}^{*}, \dot{\mathrm{s}}^{*}\right) .
$$

It follows that we can always find $\ddot{s}_{1}$ and $\ddot{s}_{2}$ such that

$$
\operatorname{MaAS}\left(\mathrm{s}^{*}, \dot{\mathrm{s}}^{*}\right)<\ddot{\mathrm{s}}_{2}<\ddot{\mathrm{s}}^{*}<\ddot{\mathrm{s}}_{1}<\operatorname{MaAS}\left(\mathrm{s}^{*}, \dot{\mathrm{s}}^{*}\right) \text {. }
$$

Now, by equation (7), we have the equality

$$
b_{k}\left(s^{*}\right) \dot{s}^{*} \ddot{s}^{*}+c_{k}\left(s^{*}\right) \dot{s}^{* 3}+d_{k}\left(s^{*}\right)=0,
$$

since $a_{k}\left(s^{*}\right)=0$. Now, if $b_{k}\left(s^{*}\right)>0$, we replace $\ddot{s}^{*}$ with $\ddot{s}_{1}$ and notice that $\dot{s}>0$ to see that equation (11) is strictly positive. Similarly if $b_{k}\left(s^{*}\right)<0$ then we replace $\ddot{s}^{*}$ with $\ddot{s}_{2}$ to see that equation (11) is strictly negative. Both are contradictions. We neglect the case where $b_{k}\left(s^{*}\right)=0$.

Finally, to prove the second proposition, we simply remark that if $b_{k}\left(s^{*}\right)>0$, then there must not exists any feasible $\ddot{s}_{1}$ that is greater than $\ddot{s}^{*}$. It therefore follows that

$$
\ddot{s}^{*}=\operatorname{MaAS}\left(\mathrm{s}^{*}, \dot{\mathrm{s}}^{*}\right) \text {. }
$$

The case where $b_{k}\left(s^{*}\right)<0$ is proven similarly.

Proposition 2. The maximum and minimum jerks are equal almost everywhere on the MaAS and the MiAS except on singular curves.

Proof. Considering a point at $\left(s_{0}, \dot{s}_{0}, \ddot{s}_{0}\right)$ on the MaAS which does not lie on any singular curve, by definition, $\ddot{s}_{0}$ is the maximum acceleration for $s=s_{0}, \dot{s}=\dot{s}_{0}$ subjecting to constraints (7). Since all constraints (7) are linear the set of feasible $(\ddot{s}, \dddot{s})$ is a convex polygon on the plane.

Now, since this polygon does not contain any edge that is parallel to the $\ddot{s}$-axis $\left(\left(s_{0}, \dot{s}_{0}, \ddot{s}_{0}\right)\right.$ does not lie on any singular curve), $\ddot{s}=\ddot{s}_{0}$ is maximized at a single vertex of the polygon. Therefore there is only one feasible jerk for $\ddot{s}=\ddot{s}_{0}$.

A similar proof can be written for the MiAS.

\section{Proposition 3. Singular curves are connected.}

Proof. Consider a singular curve $C$ in the $(s, \dot{s}, \ddot{s})$ space triggered by the $k$-th constraint. Let the convex set defined by the feasibility condition (7) be $\mathcal{A}$ and the mapping from $\mathcal{A}$ using the equality

$$
b_{k}\left(s^{*}\right) \ddot{s} \ddot{s}+c_{k}\left(s^{*}\right) \dot{s}^{3}+d_{k}\left(s^{*}\right)=0 .
$$

to the singular curve $C$ be $f$. By definition, $f(\mathcal{A})=C$.

Now, since $\mathcal{A}$ is convex and thus connected and $f$ is also continuous for $b_{k}\left(s^{*}\right) \neq 0, \dot{s}^{*} \neq 0$, using Theorem 4.22 in [23], it follows that $C$ is connected.

\section{REFERENCES}

[1] J. Bobrow, S. Dubowsky, and J. Gibson, "Time-optimal control of robotic manipulators along specified paths," The International Journal of Robotics Research, vol. 4, no. 3, pp. 3-17, 1985.

[2] K. Shin and N. McKay, "Selection of near-minimum time geometric paths for robotic manipulators," IEEE Transactions on Automatic Control, vol. 31, no. 6, pp. 501-511, 1986.
[3] Q.-C. Pham, "A general, fast, and robust implementation of the time-optimal path parameterization algorithm," IEEE Transactions on Robotics, vol. 30, pp. 1533-1540, 2014.

[4] Z. Shiller and H. Lu, "Computation of path constrained time optimal motions with dynamic singularities," Journal of dynamic systems, measurement, and control, vol. 114, p. 34, 1992.

[5] Z. Shiller and Y. Gwo, "Dynamic motion planning of autonomous vehicles," IEEE Transactions on Robotics and Automation, vol. 7, no. 2, pp. 241-249, 1991.

[6] Q.-C. Pham and Y. Nakamura, "Time-optimal path parameterization for critically dynamic motions of humanoid robots," in IEEE-RAS International Conference on Humanoid Robots, 2012, pp. 165-170.

[7] Q.-C. Pham and O. Stasse, "Time-optimal path parameterization for redundantly actuated robots: A numerical integration approach," Mechatronics, IEEE/ASME Transactions on, vol. 20, no. 6, pp. 3257-3263, 2015.

[8] S. Caron, Q.-C. Pham, and Y. Nakamura, "Leveraging cone double description for multi-contact stability of humanoids with applications to statics and dynamics," Robotics: Science and System, 2015.

[9] Q.-C. Pham, S. Caron, P. Lertkultanon, and Y. Nakamura, "Admissible velocity propagation: Beyond quasi-static path planning for highdimensional robots," The International Journal of Robotics Research, vol. 36, no. 1, pp. 44-67, 2017.

[10] M. Tarkiainen and Z. Shiller, "Time optimal motions of manipulators with actuator dynamics," in Robotics and Automation, 1993. Proceedings., 1993 IEEE International Conference on. IEEE, 1993, pp. 725730.

[11] J. Stoer and R. Bulirsch, Introduction to numerical analysis. Springer Science \& Business Media, 2013, vol. 12.

[12] J. Mattmüller and D. Gisler, "Calculating a near time-optimal jerkconstrained trajectory along a specified smooth path," The International Journal of Advanced Manufacturing Technology, vol. 45, no. 9-10, pp. 1007-1016, 2009.

[13] D. Verscheure, B. Demeulenaere, J. Swevers, J. De Schutter, and M. Diehl, "Practical time-optimal trajectory planning for robots: a convex optimization approach," IEEE Transactions on Automatic Control, 2008.

[14] K. Hauser, "Fast interpolation and time-optimization with contact," The International Journal of Robotics Research, vol. 33, no. 9, pp. 12311250, 2014.

[15] Q. Zhang, S.-R. Li, and X.-S. Gao, "Practical smooth minimum time trajectory planning for path following robotic manipulators," in American Control Conference (ACC), 2013. IEEE, 2013, pp. 2778-2783.

[16] A. Gasparetto and V. Zanotto, "A technique for time-jerk optimal planning of robot trajectories," Robotics and Computer-Integrated Manufacturing, vol. 24, no. 3, pp. 415-426, 2008.

[17] A. K. Singh and K. M. Krishna, "A class of non-linear time scaling functions for smooth time optimal control along specified paths," in Intelligent Robots and Systems (IROS), 2015 IEEE/RSJ International Conference on. IEEE, 2015, pp. 5809-5816.

[18] D. Costantinescu and E. A. Croft, "Smooth and time-optimal trajectory planning for industrial manipulators along specified paths," Journal of robotic systems, vol. 17, no. 5, pp. 233-249, 2000.

[19] A. Bharathi and J. Dong, "A Smooth Trajectory Generation Algorithm for Addressing Higher-Order Dynamic Constraints in Nanopositioning Systems," Procedia Manufacturing, vol. 1, pp. 216-225, 2015.

[20] L. Liu, C. Chen, X. Zhao, and Y. Li, "Smooth Trajectory Planning for a Parallel Manipulator with Joint Friction and Jerk Constraints," International Journal of Control, Automation and Systems, pp. 1-15, 2016.

[21] F. Pfeiffer and R. Johanni, "A concept for manipulator trajectory planning," Robotics and Automation, IEEE Journal of, vol. 3, no. 2, pp. 115-123, 1987.

[22] Z. Shiller and H.-H. Lu, "Computation of path constrained time optimal motions with dynamic singularities," Journal of dynamic systems, measurement, and control, vol. 114, no. 1, pp. 34-40, 1992.

[23] W. Rudin, Principles of mathematical analysis. McGraw-Hill New York, 1964, vol. 3. 